\title{
1,2,4-Triazole Complexes VIII *
}

Magnetic properties of cobalt(II) (1,2,4-triazole $)_{2}(\mathrm{NCS})_{2}$, a quasi two-dimensional canted $S=\frac{1}{2}$ antiferromagnet, with XY-type anisotropy

D. W. Engelfriet, W. L. Groeneveld,

Dept. Inorganic Chemistry, Gorlaeus Laboratoria, Rijksuniversiteit, Leiden, The Netherlands,

H. A. Groenendijk, J. J. Smit, and G. M. Nap

Kamerlingh Onnes Laboratorium, Rijksuniversiteit, Leiden, The Netherlands

Z. Naturforsch. 35 a, 115-128 (1980) ; received November 22, 1979

$\mathrm{Co}(\mathrm{trz})_{2}(\mathrm{NCS})_{2}$ has been investigated by means of magnetic susceptibility and magnetization measurements on single crystals in the temperature range from $1.1-300 \mathrm{~K}$, heat capacity measurements from 1.5-90 K, neutron powder diffraction at $1.2 \mathrm{~K}$ and ESR measurements on the Codoped $\mathrm{Zn}$ compound at $9 \mathrm{~K}$. The compound appears to have two-dimensional structural and magnetic properties. A transition to an antiferromagnetically ordered state takes place at $T_{\mathrm{c}}=5.71(1) \mathrm{K}$. Susceptibility measurements along the three orthorhombic axes reveal a strongly anisotropic character. The $b$ axis is found to be the preferred axis of antiferromagnetic alignment. The susceptibility data along $b$ and the heat capacity results are in good agreement with the predictions for the quadratic layer, $\mathrm{S}=\frac{1}{2}, \mathrm{XY}$ antiferromagnetic, with an intralayer exchange constant $J / k=-5.0(1) \mathrm{K}$. Below $T_{\mathrm{c}}$ the field dependent behaviour of the magnetization and the susceptibility along the $a$ axis exhibits a transition at $\sim 1 \mathrm{kOe}$. Hidden canting is found to be present. The magnetic structure can be described by four sublattices with the magnetic moments lying in the $a \cdot b$ plane along directions that are at an angle of $7^{\circ}$ with the $b$ axis.

\section{Introduction}

Properties characteristic of low-dimensional magnetic behaviour can in many compounds be related to their crystal structures. Well known 2 -d $(d=d$ mensional) magnetic systems are e.g. compounds with the $\mathrm{K}_{2} \mathrm{NiF}_{4}$ and $\mathrm{BaNiF}_{4}$ structures and compounds of composition $\left(\mathrm{C}_{n} \mathrm{H}_{2 n+1} \mathrm{NH}_{3}\right){ }_{2} \mathrm{M}(\mathrm{II}) \mathrm{X}_{4}$, with $n=0,1,2, \ldots, \mathrm{M}$ is a $3 \mathrm{~d}$-metal and $\mathrm{X}=\mathrm{Cl}$, $\mathrm{Br}$. I. A review of the magnetic properties of these layered structures has been given by De Jongh and Miedema [1]. In the above-mentioned compounds the layers are formed by edge-sharing $\mathrm{MX}_{6}$ octahedra. The strongest exchange takes place via the halide ions, connecting each metal ion to four nearest neighbouring metal ions. The layers are well separated by non-magnetic atoms, so that the exchange between metal ions in neighbouring layers is several orders of magnitude smaller than the intralayer exchange. The number of known 2-d compounds in which intralayer exchange takes place via ligands consisting of more than one atom is much smaller. Some examples are: M(II) $(\mathrm{HCOO})_{2} \cdot 2 \mathrm{H}_{2} \mathrm{O}$, with $\mathrm{M}=\mathrm{Mn}, \mathrm{Fe}$, Co or $\mathrm{Ni}, \mathrm{Cu}(\mathrm{HCOO})_{2} \cdot 4 \mathrm{H}_{2} \mathrm{O}$ [1] and $\mathrm{Mn}\left(\mathrm{C}_{2} \mathrm{H}_{5} \mathrm{OH}\right)_{2}(\mathrm{NCS})_{2}$ [2].

\footnotetext{
* Part VII : J. G. Haasnoot and W. L. Groeneveld, Z. Naturforsch. submitted.

Requests for reprints should be sent to Drs. D. W. Engelfriet, Gorlaeus Laboratoria, P.O. Box 95 02, 2300 RA Leiden, The Netherlands.
}

As regards complexes with nitrogen-heterocyclic bridging ligands, an extensive review on the magnetic interactions in such complexes was given by Inoue and Kubo [3]. They mentioned only one group of compounds which from their structure might be considered for 2-d magnetic behaviour, namely $\mathrm{M}$ (II) (pyrazine) ${ }_{2} \mathrm{X}_{2}$. The structure of the tetragonal $\mathrm{Co}(\mathrm{pyz})_{2} \mathrm{Cl}_{2}$ was found to consist of quadratic layers of $\mathrm{Co}$ (II) ions bridged in the planes by pyrazine groups, with $\mathrm{Cl}^{-}$ions on either side of the planes [4]. However, down to $1.8 \mathrm{~K}$ no ordering was found [3] and it was shown recently that this compound behaves as a 3-d XY antiferromagnet with $J / k=-0.42 \mathrm{~K}$ and $T_{\mathrm{c}}=0.855 \mathrm{~K}$ [5].

A layered crystal structure has also been found in the recently discovered series of compounds $\mathrm{M}$ (II) $(\operatorname{trz})_{2}(\mathrm{NCS})_{2}$ [6] (M is a 3d-metal and trz is $1,2,4$ triazole). These compounds exhibit 2-d properties both structurally and magnetically, in contrast with $\mathrm{Co}(\mathrm{pyz}){ }_{2} \mathrm{Cl}_{2}$. In a previous report we showed that the magnetic behaviour of $\mathrm{Mn}(\operatorname{trz})_{2}(\mathrm{NCS})_{2}$ could be accounted for by considering this material to be a 2-d, $S=5 / 2$ Heisenberg antiferromagnet [9]. By means of X-ray powder diffraction the Mn complex was found to be isomorphous to the corresponding Co one, the structure of which has been determined by X-ray single crystal diffraction [8]. Susceptibility measurements on powdered $\mathrm{Co}(\operatorname{trz})_{2}(\mathrm{NCS})_{2}$ revealed a large peak at $5.9 \mathrm{~K}$ and in the magnetiza-

0340-4811 / $80 / 0100-0115 \$ 01.00 / 0$. - Please order a reprint rather than making your own copy. 
tion vs. field curve a distinct jump was found at $1 \mathrm{kOe}$ [7]. These results were ascribed to the presence of canting, associated with the tilted $\mathrm{CoN}_{6}$ octahedra in the crystal structure. The jump in the magnetization curve is reminiscent of the behaviour observed in e. g. Co (pyridine) ${ }_{2} \mathrm{Cl}_{2}$ and related compounds [10] and in $\mathrm{CsCoCl}_{3} \cdot 2 \mathrm{H}_{2} \mathrm{O}[11,12]$.

In this paper we report our investigations of the magnetic properties of $\mathrm{Co}(\operatorname{trz})_{2}(\mathrm{NCS})_{2}$ by means of susceptibility and magnetization measurements on single crystals, neutron diffraction experiments and heat capacity measurements on powdered samples and by ESR measurements on Co doped $\mathrm{Zn}(\operatorname{trz})_{2}(\mathrm{NCS})_{2}$.

\section{Crystal Structure}

$\mathrm{Co}(\operatorname{trz})_{2}(\mathrm{NCS})_{2}$ is orthorhombic, space group $\mathrm{Aba} 2$, with $a=7.801, b=16.331, c=9.738 \AA$ and $Z=4$. The structure is presented in Figure 1 . The Co(II) ions occupy special positions $(0,0,0)$ and $\left(0, \frac{1}{2}, \frac{1}{2}\right)$. They are octahedrally coordinated by two $N(2)$ and two $N(4)$ atoms from triazole groups and by two nitrogen atoms from $\mathrm{NCS}^{-}$groups. The $\mathrm{CoN}_{6}$ octahedra are tilted in the $a-b$ plane, the angle with the $b$ direction being $31.0^{\circ}$. The planar trz groups make an angle of $0.2^{\circ}$ with the $b$ direction and by coordinating via their $\mathrm{N}(2)$ and $\mathrm{N}(4)$ atoms, a 2-d layered structure is formed. The strongest exchange between Co ions will take place via the bridging trz groups in the planes $y=0$ and $y$ $=\frac{1}{2}$. Thus each Co ion is connected equivalently to four nearest neighbours and the planes $y=0$ and $y=\frac{1}{2}$ can be considered as quasi-quadratic magnetic lattices.

Fairly short distances are found between an $\mathrm{S}$ atom in one layer and $\mathrm{H}(1)$ and $\mathrm{H}(5)$ atoms belonging to a trz ring in a neighbouring layer, suggesting the presence of hydrogen bonding [8]. As carbon atoms usually do not act as hydrogen bond donors, the strongest interlayer coupling will most probably be formed by the NCS ...H(1) - N(1) bond. Thus, interlayer exchange can take place via the exchange path $\mathrm{Co}-\mathrm{N}-\mathrm{C}-\mathrm{S} \ldots \mathrm{H}(1)-\mathrm{N}(1)-\mathrm{N}(2)-$ Co. From the large number of non-magnetic atoms involved in this bridge the interlayer interaction is expected to be much weaker than the intralayer exchange, which explains the observed 2-d character.

It should be mentioned here that Fig. 1 represents a simplified picture of the structure. In the refinement of the X-ray diffraction data it was found that disorder in the trz rings is present. This disorder implies that $50 \%$ of the trz rings is rotated over $180^{\circ}$ around an axis pointing from $(\mathrm{C}(3)$ to halfway between $\mathrm{C}(5)$ and $\mathrm{N}(1)$. A similar picture
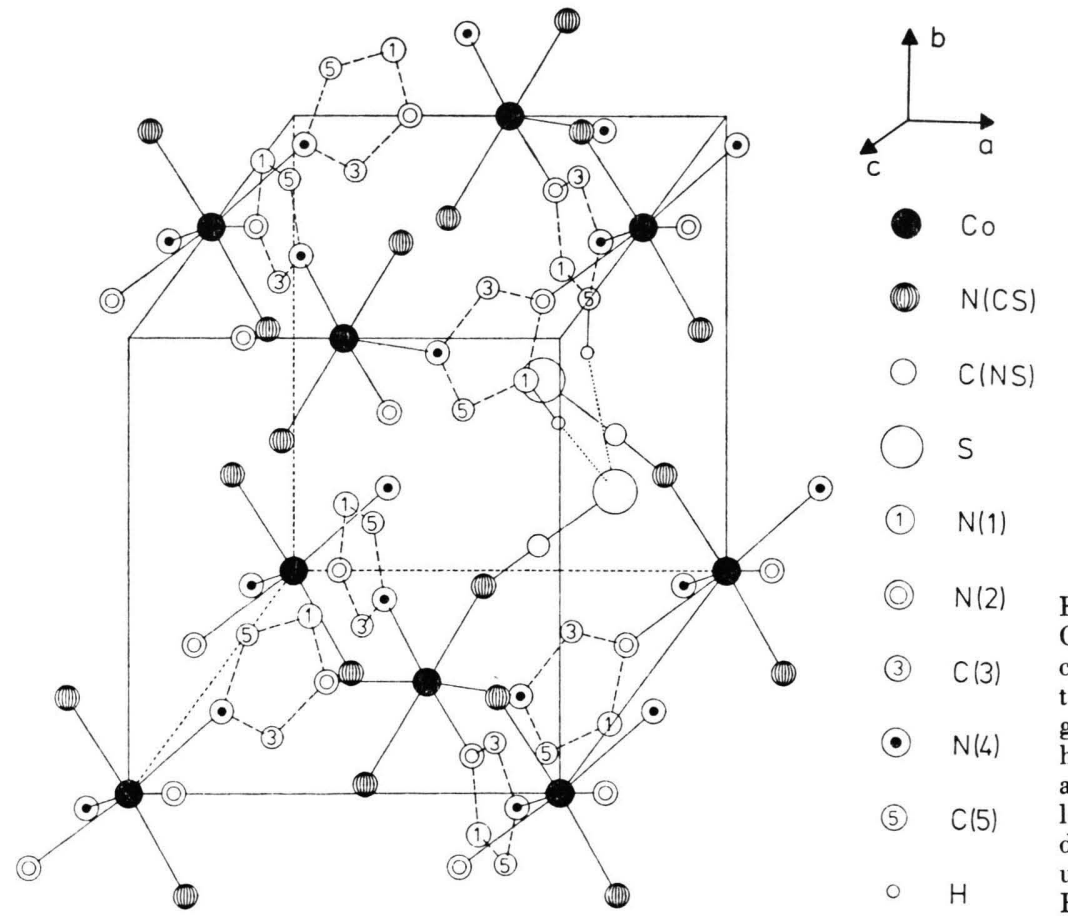

Fig. 1. The crystal structure of $\mathrm{Co}(\mathrm{trz})_{2}(\mathrm{NCS})_{2}$. The top half of the unit cell has been omitted. For clarity only two isothiocyanate groups and two hydrogen atoms are shown. The numbers of the hydrogen atoms are those of the ring atoms they are attached to. Possible interlayer hydrogen bonding is indicated by dotted lines. This figure has been generated using the crystallographic plotting program FIGATOM [13]. 
emerged from X-ray diffraction experiments on a $\mathrm{Fe}(\mathrm{trz})_{2}(\mathrm{NCS})_{2}$ single crystal [14].

Whether there is disorder throughout the structure or that domains are present in which the trz rings are reversed could not be established from the X-ray data. However, effects resulting from this disorder are not expected to occur in our magnetic measurements.

\section{Experimental}

Heat capacity measurements were performed in the temperature range between 1.5 and $90 \mathrm{~K}$ using conventional heat pulse techniques. The powdered sample (mass about $8 \mathrm{~g}$ ), which was prepared according to the method given by Haasnoot and Groeneveld [6], was compressed into a calorimeter can. The apparatus and measuring procedures have been described elsewhere [15]. Single crystals of $\mathrm{Co}(\operatorname{trz})_{2}(\mathrm{NCS})_{2}$ were grown by slow evaporation at about $70^{\circ} \mathrm{C}$ of an acidified solution of the complex. Orange-red crystals which are frequently intergrown are formed in this way. They are usually quite flat and diamond-shaped, though the diamond is sometimes elongated to a parallelogram. From X-ray experiments it was established that the $b$ axis is perpendicular to the flat surface of the crystals and that the diamond shape corresponds with the pattern of the Co ions in the $y=0$ and $y=\frac{1}{2}$ planes as was also found for $\mathrm{Mn}(\operatorname{trz})_{2}(\mathrm{NCS})_{2}$ [9]. The $a$ and $c$ axes are along the bisectors of the angles of the diamond or parallelogram. Several crystals with a total mass of about $30 \mathrm{mg}$ were mounted with Apiezon grease into a small delrin cube, which was fixed to the sample rod of a commercial vibrating sample magnetometer, equipped with a superconducting magnet supplying fields up to $56 \mathrm{kOe}$ [16]. Measurements from $80-300 \mathrm{~K}$ were taken using the Faraday method [17]. Since in the Faraday set-up the sample could not be orientated rigidly, reliable results could only be obtained in the $b$ and $c$ directions, due to the strong anisotropy in the $a-b$ and $a-c$ planes (see below $)$. The a.c. susceptibility $(v=0.5 \mathrm{~Hz}-$ $10 \mathrm{kHz}$ ) in the $a$ direction was measured in the temperature range from $2-20 \mathrm{~K}$ using the mutual inductance technique [18]. The measurements were made both in zero-field and in constant fields up to $4.2 \mathrm{kOe}$. These measurements were performed on a single crystal of $29.0 \mathrm{mg}$. Magnetization measurements up to $300 \mathrm{kOe}$ were carried out in pulsed mag- netic fields [19] along the $a$ and $b$ directions on samples of about $125 \mathrm{mg}$, composed of a number of small single crystals.

All measured susceptibilities and magnetizations were corrected for diamagnetism using a value of $-156(5) \times 10^{-6}$ e.m.u./mole as we determined for the $\mathrm{Zn}$ compound.

ESR measurements were performed with a commercial X-band spectrometer fitted with a helium flow-cryostat.

The neutron diffraction pattern was recorded using the powder diffractometer at the HFR reactor at Petten.

\section{Results}

\section{Heat capacity measurements}

Figure 2 shows the measured specific heat of $\mathrm{Co}(\mathrm{trz})_{2}(\mathrm{NCS})_{2}$ as a function of temperature. From the temperature where the peak occurs the ordering temperature is found as $T_{\mathrm{c}}=5.71(1) \mathrm{K}$ [20]. To obtain the magnetic heat capacity $\left(C_{\mathrm{M}}\right)$ the lattice heat capacity $\left(C_{\mathrm{L}}\right)$ has to be subtracted from the experimental data. The fairly low value of $T_{\mathrm{c}}$ suggests that this may be possible by finding a temperature region where the total specific heat can be given as $C=C_{\mathrm{M}}+C_{\mathrm{L}}=b T^{-2}+a T^{3}$. However, such a temperature region could not be found. Therefore, assuming the corresponding states procedure [21] to be valid, we tried to determine the lattice contribution by using the heat capacity data of the corresponding $\mathrm{Mn}(\mathrm{II})$ and $\mathrm{Cu}(\mathrm{II})$ compounds. For these compounds the lattice contribution could be determined sufficiently accurate from $C T^{2}$ vs $T^{5}$ plots, since the values of $T_{\mathrm{c}}$ are lower $\left(T_{\mathrm{c}}=3.29 \mathrm{~K}\right.$ and $0.7 \mathrm{~K}$ for the $\mathrm{Mn}(\mathrm{II})$ and $\mathrm{Cu}(\mathrm{II})$ compounds, respectively). In the fitting procedure it became clear that the $\mathrm{Co}$ (II) ions are in a doublet ground state with an effective $S=\frac{1}{2}$ and that for $T \gtrsim 20 \mathrm{~K}$ there are contributions to the specific heat due to higher electronic levels. A satisfactory result was obtained with $C_{\mathrm{L}, \mathrm{Co}}(T)=C_{\mathrm{L}, \mathrm{Cu}}(T / 1.111)$, which is the dashed line in Figure 2. The magnetic specific heat for $T<20 \mathrm{~K}$ as a function of temperature is shown in Fig. 3 in a double logarithmic plot. In order to determine the total magnetic entropy $\Delta S$ and energy $\Delta E$ the curve was extrapolated to $T=\infty$. Contributions below $T=1.5 \mathrm{~K}$ amount to less than $1 \%$ and can be neglected. In this way we found $\Delta S /$ $R=0.69(3) \mathrm{K}$ which agrees within experimental 


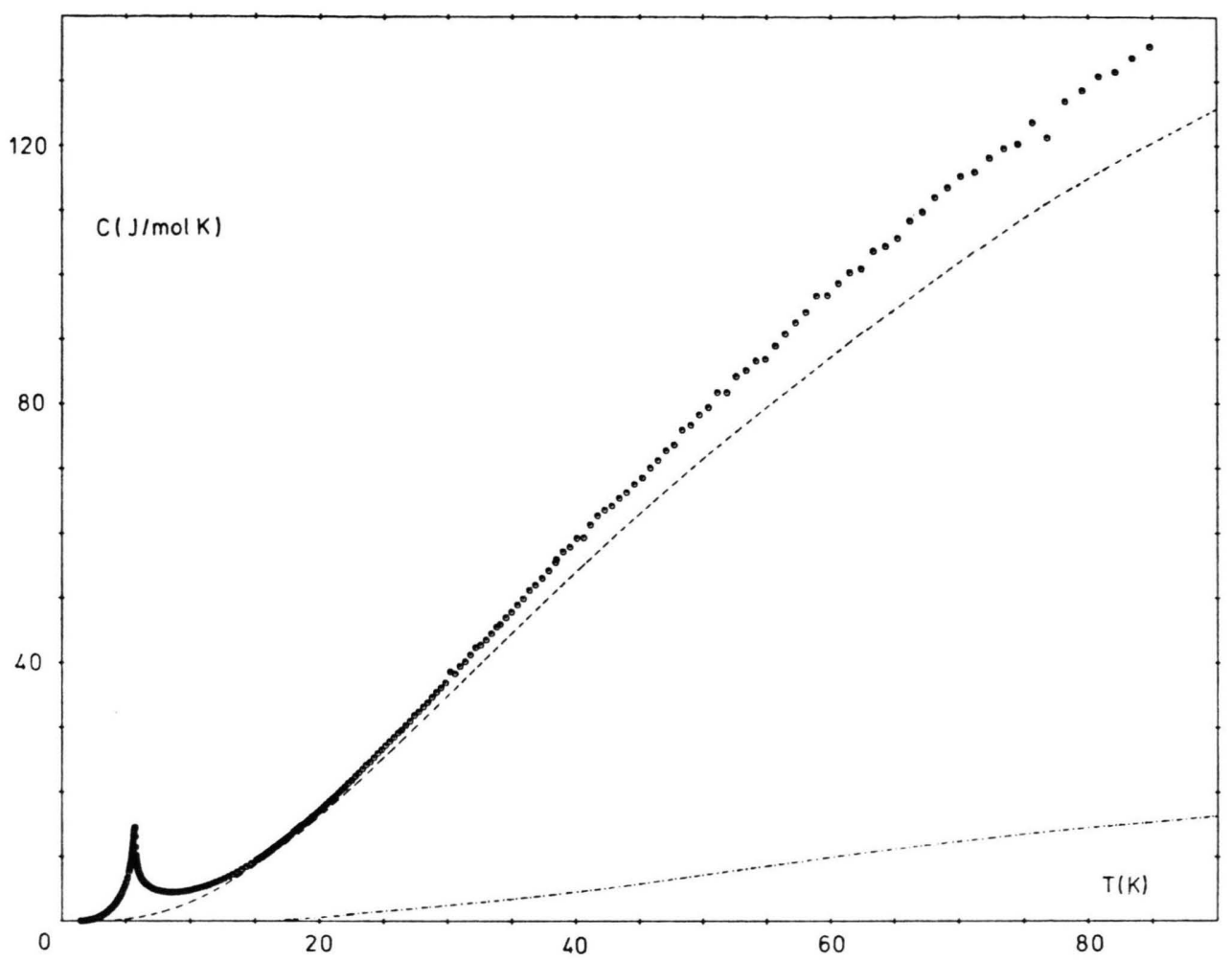

Fig. 2. The heat capacity as a function of temperature. The dashed line is the lattice contribution, the dash-dotted line is the excess heat capacity, obtained by subtraction of the lattice and magnetic contributions from the total heat capacity.

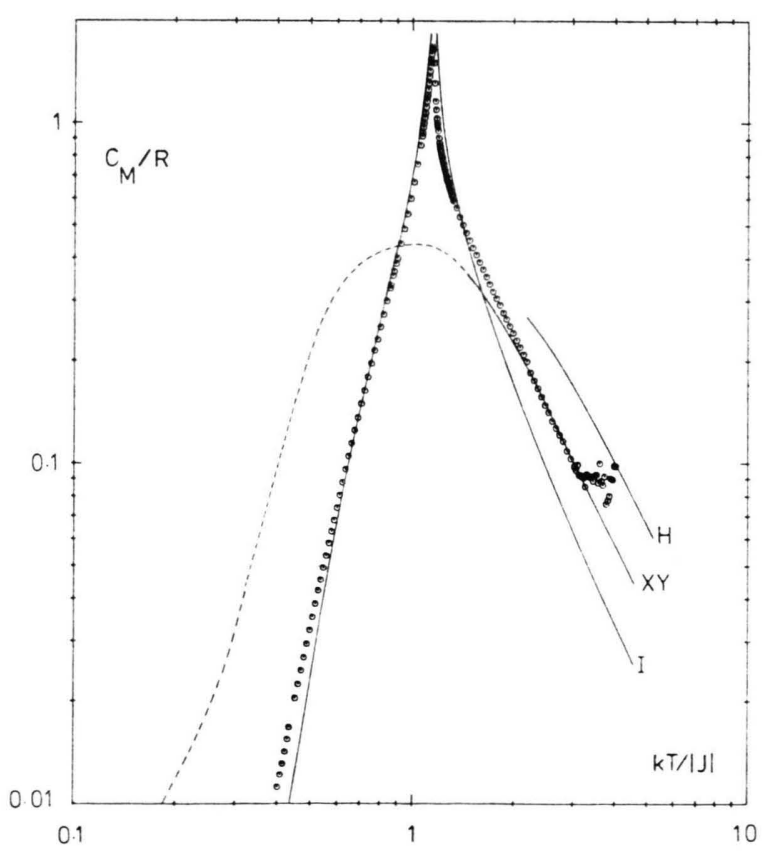

Fig. 3. The magnetic heat capacity compared with the predictions for the Ising (I), XY and Heisenberg (H) models, all for the $S=\frac{1}{2}$ quadratic lattice. The data have been scaled on the high-temperature part of the XY curve by taking $\mathrm{J} / k=5.0 \mathrm{~K}$. error with the theoretical value for $S=\frac{1}{2}(0.693 \mathrm{~K})$. The entropy below $T_{\mathrm{c}}$ amounts to $42 \%$ of the total entropy gain. For the total magnetic energy $\Delta E / R$ $=5.8(3) \mathrm{K}$ is obtained, of which $24 \%$ is gained below $T_{\mathrm{c}}$. The dash-dotted line in Fig. 2 represents the excess heat capacity, obtained after subtracting the above magnetic and lattice contributions from the total heat capacity. Although the excess specific heat shows that there are contributions from higher energy levels, its value of $\sim 17 \mathrm{~J} / \mathrm{mol} \mathrm{K}$ at $90 \mathrm{~K}$ is much higher than one would expect for a Schottkytype of anomaly. Apparently, the correct lattice heat capacity of $\mathrm{Co}(\operatorname{trz})_{2}(\mathrm{NCS})_{2}$ at higher temperatures can not be obtained from the heat capacity of $\mathrm{Cu}(\operatorname{trz})_{2}(\mathrm{NCS})_{2}$ by means of a temperature independent scaling factor.

\section{Susceptibility and magnetization measurements}

Figure 4 shows the static susceptibility as derived from the magnetization measurements along the $b$ and $c$ directions. Broad maxima are found in the susceptibility curves which are an indication for lowdimensional behaviour. The $b$ axis is the easy axis of antiferromagnetic alignment and the $c$ axis is a perpendicular direction. The maximum in the $\chi_{\mathrm{b}}$ vs $T$ 


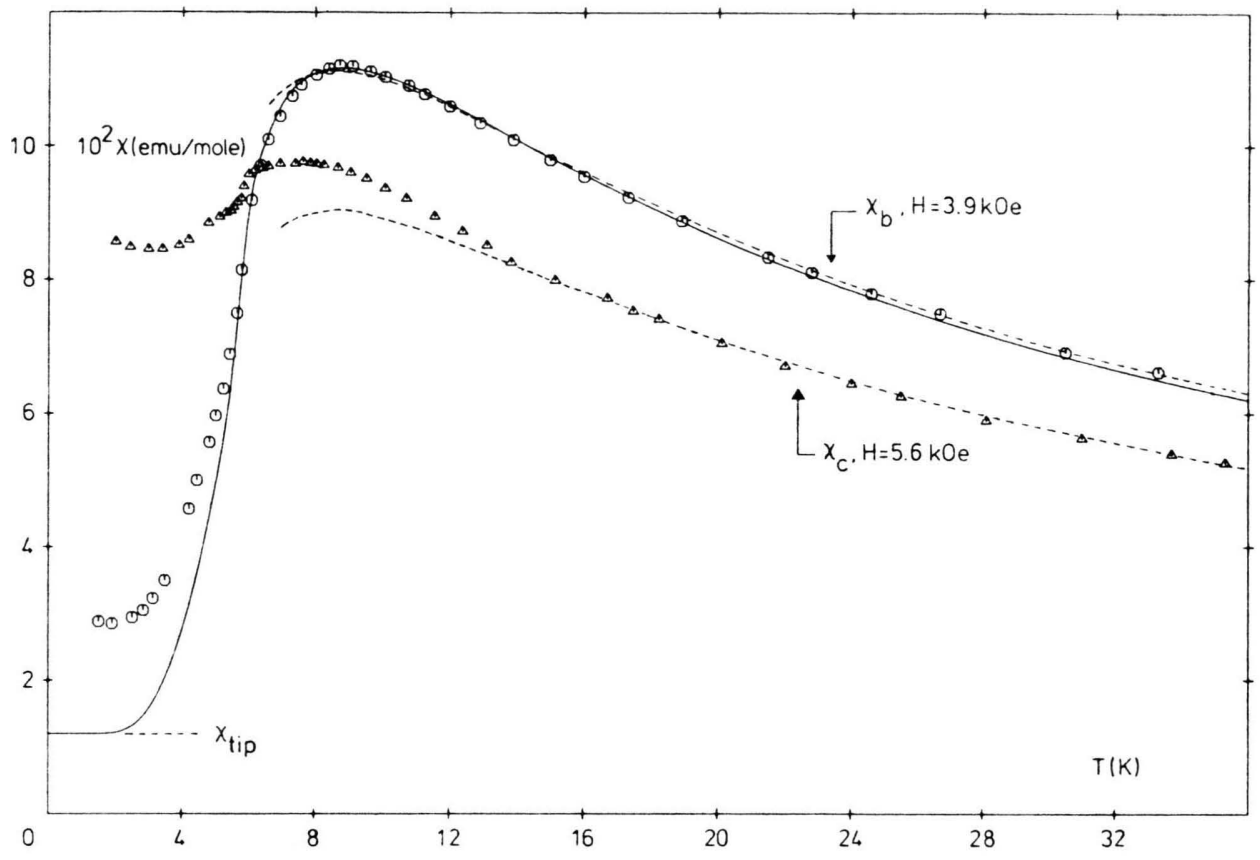

Fig. 4. Magnetic susceptibility as a function of temperature measured along the $b$ and $c$ axes. The full line represents the prediction for a 2 -d, $S=\frac{1}{2}$, Ising antiferromagnet with $J / k=-5.05 \mathrm{~K}$ and the dashed lines those for the 2 -d XY, $S=\frac{1}{2}$, antiferromagnet with $J / k=-5.0 \mathrm{~K}$.

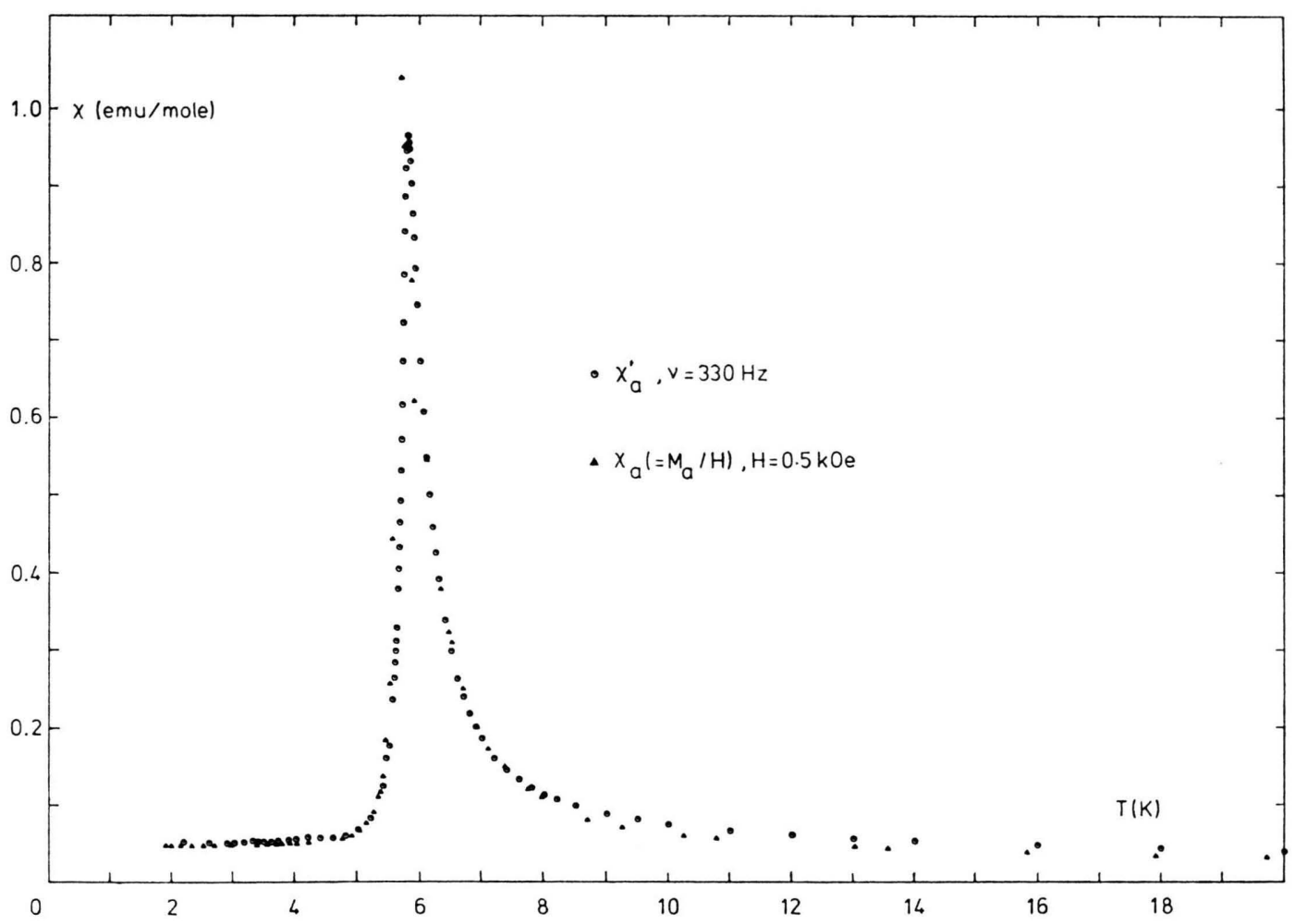

Fig. 5. Magnetization and a.c. susceptibility as a function of temperature measured along the $a$ axis. 
curve occurs at $8.8(1) \mathrm{K}$, with $\chi_{\mathrm{b}}(\max )=$ $0.112(2)$ e.m.u./mole. The $\chi_{\mathrm{c}}$ vs $T$ curve has a less clearly defined maximum at $T=7.5(5) \mathrm{K}$ with $\chi_{\mathrm{c}}(\max )=0.097(2)$ e.m.u./mole. From the points of maximum slope of these curves $T_{\mathrm{c}}$ can be estimated as $5.8(1) \mathrm{K}$, in agreement with the value obtained from the heat capacity measurements. Figure 5 shows the results of both magnetization and a.c. susceptibility measurements $(v=330 \mathrm{~Hz})$ in the $a$ direction. A sharp peak occurs at $5.80 \mathrm{~K}$. From the point of maximum slope of the $\chi_{\mathrm{a}}$ vs $T$ curve $T_{\mathrm{c}}=$ $5.74(1) \mathrm{K}$ is found, slightly higher than the value resulting from heat capacity measurements.

Below $T_{\mathrm{c}}, \chi_{\mathrm{a}}$ is strongly field dependent and has a sharp peak at a critical field $H_{\mathrm{c}}{ }^{\mathrm{a}}$. Figure 6 gives the magnetic phase diagram, obtained from the field value where the peak occurred. Extrapolation to $T=0 \mathrm{~K}$ yields $H_{\mathrm{c}}{ }^{\mathrm{a}}(0)=1015(10)$ Oe. For temperatures close to $T_{\mathrm{c}}$ the field $H_{\mathrm{c}}{ }^{\mathrm{a}}$ was taken as the point of maximum slope in the $\chi_{\mathrm{a}}$ vs $H$ curve. In addition to the in-phase component of the susceptibility $\chi^{\prime}$, also an out-of-phase component $\chi^{\prime \prime}$ was measured. At $5.0 \mathrm{~K}$ both $\chi_{\mathrm{a}}{ }^{\prime}$ and $\chi_{\mathrm{a}}{ }^{\prime \prime}$ have been measured at the critical field value $H_{\mathrm{c}}=906$ Oe for a number of frequencies of the a.c. field. The data were plotted in the form of a so-called Argand diagram. The diagram showed a strongly flattened semicircle, which implies that the relaxation process cannot be described by a single relaxation time [22]. The average relaxation time was found from the top of the Argand diagram as $\sim 1.5 \times 10^{-2} \mathrm{~s}$. Extrapolation to $\omega=0$ yielded a value of 11 e.m.u./mole for the isothermal susceptibility $\chi_{T}$. At lower temperatures the relaxation time at the critical field increases strongly $(\sim 10 \mathrm{~s}$ at $2 \mathrm{~K})$, so, that $\chi_{T}$ could not be determined from the a.c. susceptibility.

Figures $7 \mathrm{a}$ and $7 \mathrm{~b}$ represent the magnetization in the $a$ direction as a function of the applied field at several temperatures. The drawn line in Fig. $7 \mathrm{~b}$ was obtained from pulsed field measurements and the points are data obtained in static fields (vibrating sample magnetometer). At temperatures well below $T_{\mathrm{c}}$ the magnetization increases very slowly up to a critical field of $1 \mathrm{kOe}$, then rises steeply to $0.38 \mu_{\mathrm{B}}$ (Fig. 7 a) and increases again nearly linearly up to $56 \mathrm{kOe}$ (Figure $7 \mathrm{~b}$ ). The magnitude of the jump in the magnetization (Fig. 7 a) and the value of the critical field both decrease with increasing temper-

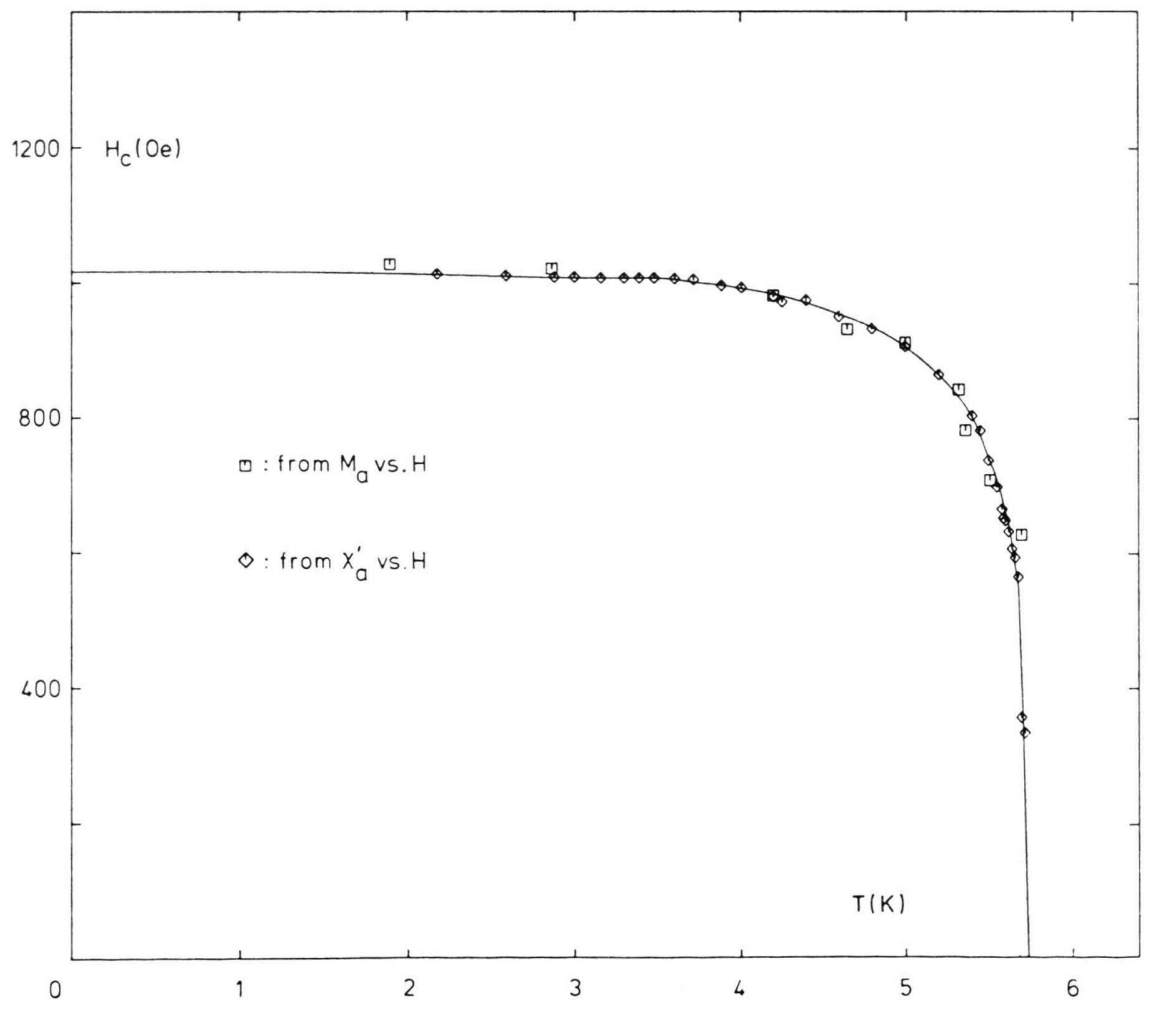

Fig. 6. Temperature dependence of the critical field $H_{\mathrm{c}}{ }^{\mathrm{a}}$, as obtained from $\chi^{\prime}$ vs $H$ and $M_{\mathrm{a}}$ vs $H$ measurements. A smooth curve is drawn through the experimental points. 

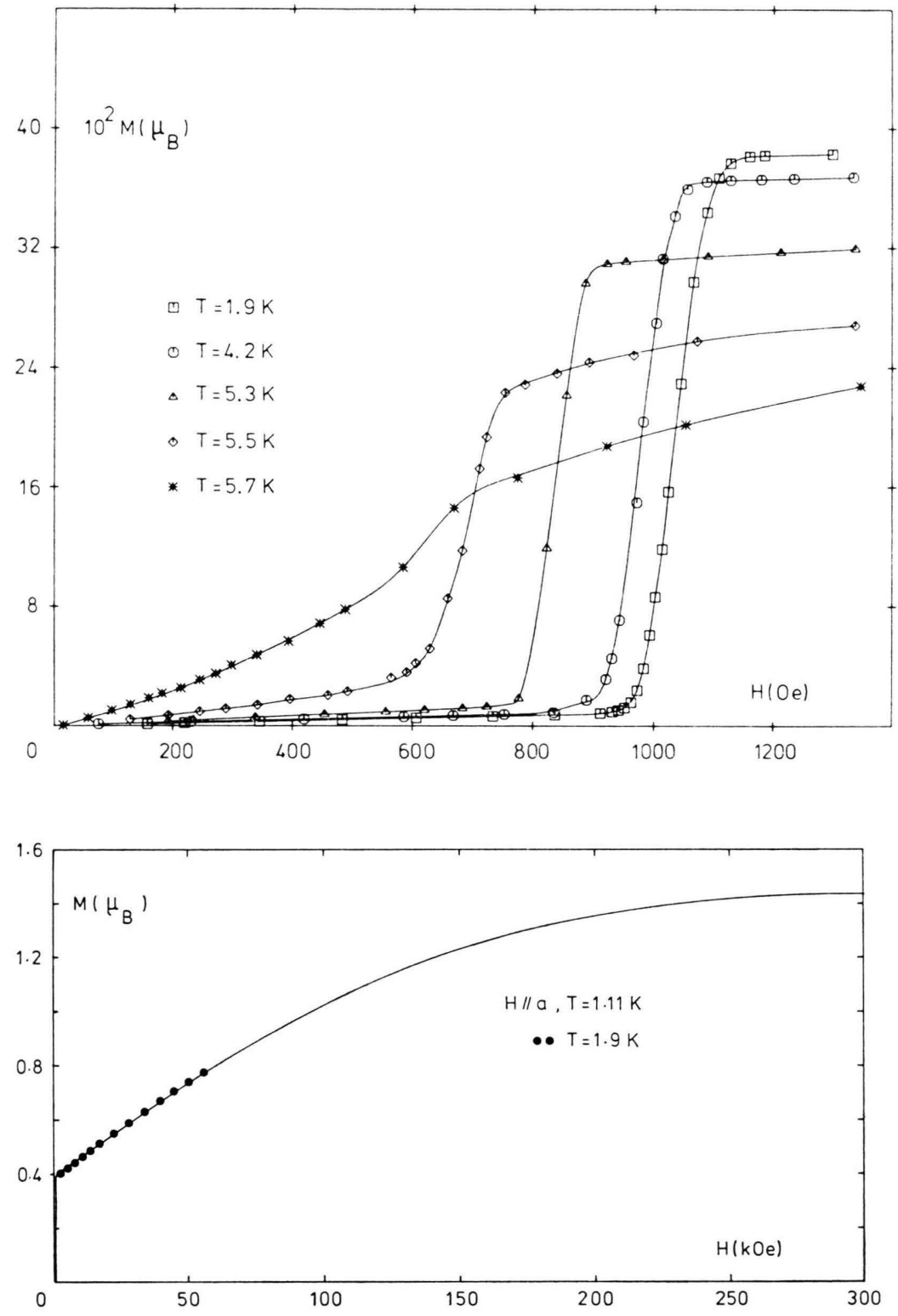

Fig. 7 a. Magnetization as a function of the applied field along the $a$ direction at some different temperatures. The data at 5.5 and $5.7 \mathrm{~K}$ were measured on a different sample. The drawn curves are for visual aid only.

Fig. 7 b. Magnetization curve measured along the $a$ direction in pulsed fields. The points represent data obtained in static fields. ature. The critical field $H_{\mathrm{c}}{ }^{\mathrm{a}}$ is taken as the point of maximum slope in the $M_{\mathrm{a}}$ vs $H$ curve. The values of $H_{\mathrm{c}}{ }^{\mathrm{a}}$ thus obtained at different temperatures have been included in the magnetic phase diagram (Figure 6). Despite the scatter in these data the values of $H_{\mathrm{c}}{ }^{\mathrm{a}}$ agree with the ones obtained from the a.c. susceptibility measurements. Saturation is reached at about $300 \mathrm{kOe}$ where $M_{\mathrm{a}}=1.45(5) \mu_{\mathrm{B}}$. Apart from the discontinuity at $1 \mathrm{kOe}$ the curve shows a gradual transition to the ferromagnetic state.
In Fig. 8 we give the results of measurements with $H / / b$. The drawn line represents the measurements in pulsed fields while the points are again obtained from the measurements in static fields. The curve displays a transition at 38(2) kOe (region of maximum slope), whereafter the magnetization increases almost linearly. This transition is reminiscent of a spin-flop transition. The transition to the ferromagnetic state takes place at a field which is estimated as $100(5) \mathrm{kOe}$. At $200 \mathrm{kOe}$ saturation is 


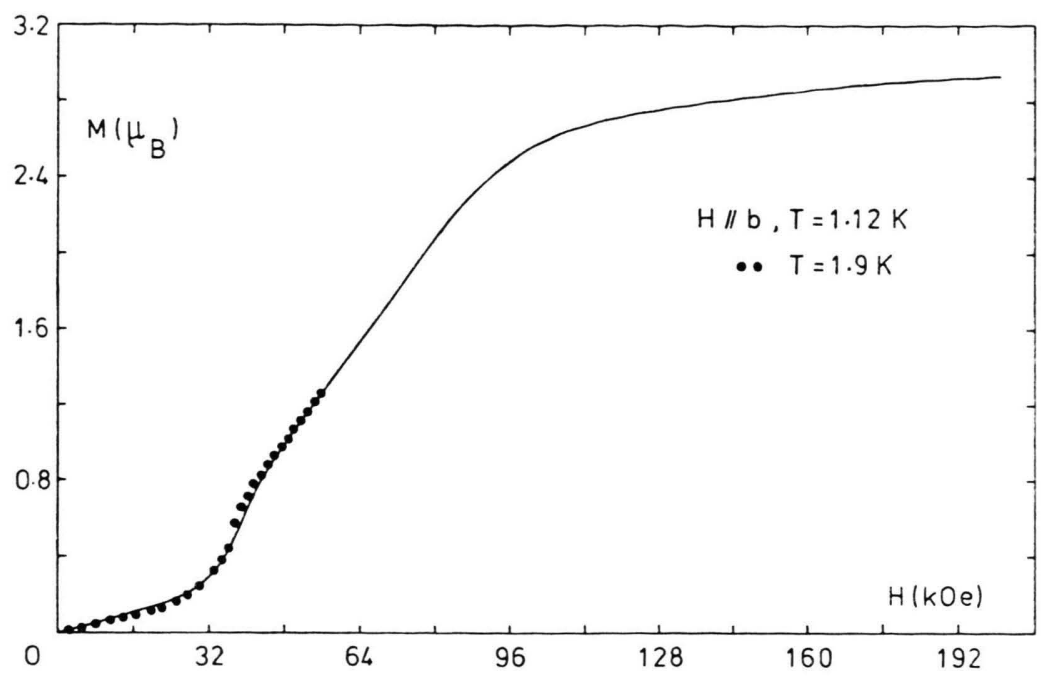

Fig. 8. Magnetization curve measured along the $b$ direction in pulsed fields. The points were obtained in static fields.

practically reached and the saturation magnetization is estimated as $2.95(5) \mu_{\mathrm{B}}$. The magnetization vs field curve in the $c$ direction yielded a straight line up to $56 \mathrm{kOe}$. No pulsed field measurements were taken along $c$.

\section{ESR measurements}

In order to obtain information on the directions of the principal axes of the $g$-tensor, ESR measurements were performed on a single crystal of $\mathrm{Zn}(\operatorname{trz})_{2}(\mathrm{NCS})_{2}$, doped with about $1 \%$ Co. The signal was recorded at $9 \mathrm{~K}$ at intervals of $10^{\circ}$, rotating the crystal around the $a, b$ and $c$ axes (with respect to the structure of $\left.\mathrm{Co}(\operatorname{trz})_{2}(\mathrm{NCS})_{2}\right)$. As may be expected from the crystal structure, two inequivalent sites could be observed, each with $g_{1}=$ $5.42(5), g_{2}=4.32(5)$, and $g_{3}=2.34(5)$. The $g_{2}$ axis is parallel to the crystallographic $c$ direction and the $g_{1}$ axes of the two sites make angles of $+10(2)^{\circ}$ and $-10(2)^{\circ}$, respectively, with the crystallographic $b$ direction.

\section{Neutron Diffraction}

The possible presence of spin canting as mentioned in the introduction made it worthwhile to investigate $\mathrm{Co}(\operatorname{trz})_{2}(\mathrm{NCS})_{2}$ by neutron diffraction below $T_{c}$, in order to determine the magnetic space group. The diffraction pattern was recorded at $1.2 \mathrm{~K}$, in the range $5.4^{\circ}<2 \theta<138^{\circ}$. The neutron wavelength was $2.5728 \AA$. Figure 9 gives the data up to the first $75^{\circ}$ in $2 \theta$. The diagram suffers from a low peak-to-background ratio, owing to the relatively high hydrogen content. Despite this shortcoming, a number of important features can be deduced from the diagram. The occurrence of reflections indexed as 001 and 110, which would be forbidden in Aba2, indicates that the magnetic lattice will be primitive. We will assume that at $1.2 \mathrm{~K}$ the nuclear frame of the lattice can still be described in $\mathrm{Aba} 2$ (ignoring disorder). In that case three possible magnetic space groups have to be considered [9, 23], namely (i) $A_{\mathrm{P}} b a^{\prime} 2$, (ii) $\mathrm{A}_{\mathrm{P}} \mathrm{ba}^{\prime} 2^{\prime}$ and (iii) $\mathrm{A}_{\mathrm{P}} \mathrm{b}^{\prime} \mathrm{a} 2^{\prime}$. Because the Co (II) ions are positioned on two-fold axes, their magnetic moments are either along $c\left(2_{z}\right.$ axis $)$ or perpendicular to $c\left(2{ }_{z}{ }^{\prime}\right.$ axis). Space group (i) can therefore be excluded, since it would restrict the spins to the $c$ direction only, which is in contradiction with the susceptibility measurements which show that the $b$ axis is the preferred direction. Possible arrangements in (ii) and (iii) are shown in Figure 10. They have tentatively been drawn in such a way, that the planes $y=0$ and $y=\frac{1}{2}$ are nearly antiferromagnetic arrays. Note that both space groups allow hidden canting and that the spins are constrained to directions perpendicular to $c$, due to the presence of a $2_{z}{ }^{\prime}$ axis. From Fig. 10 it is clear that when the $b$ axis is the preferred direction, the magnetic structure should be described by space group (iii). (It is interesting to mention here that the corresponding $\mathrm{Fe}$ (II) compound has the $a$ axis as a preferred direction [24] and may therefore be compatible with space group (ii)).

The data were further analysed quantitatively using Rietveld's profile method [25]. Atomic form 


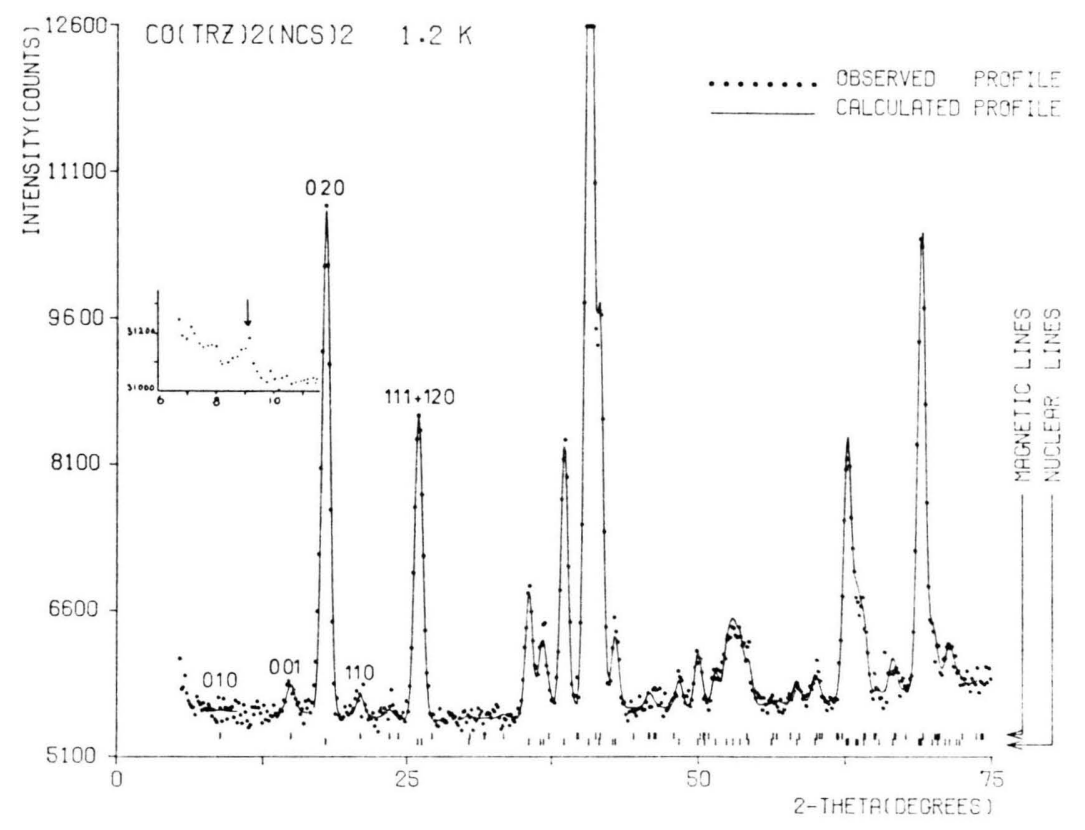

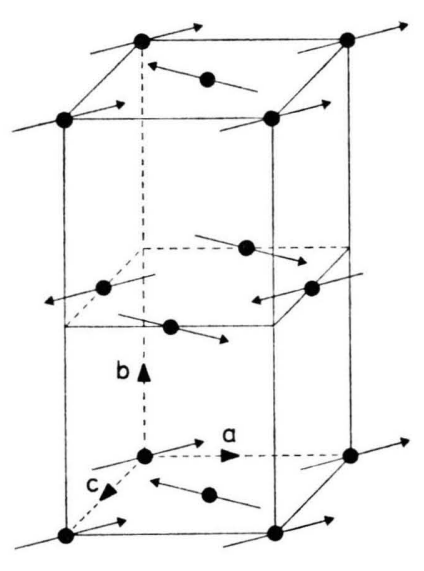

ii

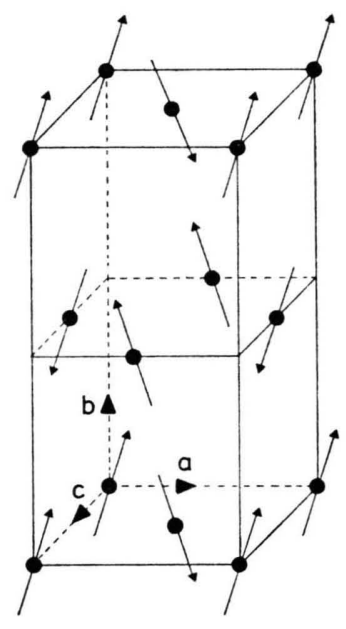

iii
Fig. 10. Possible arrangements of the magnetic moments in the space groups Apba'2'(ii) and $\mathrm{APb}^{\prime} \mathbf{2}^{\prime}$ (iii). (N. B. All magnetic moments are perpendicular to the $c$ axis due to the presence of a $2 z^{\prime}$ axis.)

factors for Co(II) were taken from Watson and Freeman [26] and scattering lengths from Bacon [27]. The residual function minimized in the leastsquares refinement is [25]:

$$
\chi^{2}=\Sigma w_{i} \mid y_{i}(\mathrm{obs})-\left.\frac{1}{c} y_{i}(\text { calc })\right|^{2} .
$$

Fig. 9. Part of the neutron diffraction pattern at $1.2 \mathrm{~K}$. Only some reflections have been labelled.
The $R$ factors (agreement indices) referred to are defined by:

$$
\begin{aligned}
& R_{\text {nuclear }}=100 \Sigma \mid I(\mathrm{obs})-\frac{1}{c} I(\text { calc }) \mid / \Sigma I(\mathrm{obs}), \\
& R_{\text {profile }}=100 \Sigma \mid y(\mathrm{obs})-\frac{1}{c} y(\text { calc }) \mid / \Sigma y(\mathrm{obs}) \\
& R_{\text {weight }} \\
& =100\left[\Sigma w \mid y(\mathrm{obs})-\left.\frac{1}{c} y(\text { calc })\right|^{2} / \Sigma w|y(\mathrm{obs})|^{2}\right]^{\frac{1}{2}} .
\end{aligned}
$$

$I$ (obs) and $I$ (calc) are the observed and calculated intensities respectively of each nuclear reflection ( $R_{\text {magnetic }}$ is defined similarly). $y$ (obs) and $y$ (calc) are the observed and calculated profile data points, $w_{i}$ is the statistical weight of a data point and $c$ is the scale factor [25]. The intensities were corrected for preferred orientation according to

$$
y_{i}(\text { corr })=y_{i}(\text { obs }) \exp \left(-G \alpha^{2}\right)
$$

where $\alpha$ is the acute angle between the scattering vector and the normal of the plate like crystallites, [010]. $G$ is known as the preferred orientation parameter. The structural parameters used in the refinement were those found by X-ray diffraction at room temperature. The drawn line in Fig. 9 represents the fit to the data, obtained after several cycles of refinement in $A_{\mathrm{P}} \mathrm{b}^{\prime} \mathrm{a}^{\prime}$, , keeping the positional parameters constant. Relevant parameters are 
listed in Table 1. The rather poor statistics of the diagram is reflected in the values of the $R$-indices and the standard deviations in $M_{x}$ and $M_{y}$, the values of the magnetization in the $x$ and $y$ directions. Refinement of atomic positions also gave lower $R$ values, but resulted in impossible distances and angles. This is not surprising, since the number of parameters is then increased with 33 . From the magnetic structure given in Fig. 10 (iii) it can be inferred that a canted spin structure requires $M_{x} \neq 0$. This would imply that the intensity of the reflection 010, being proportional to $M_{x}{ }^{2}$, should be non zero. In an attempt to observe this reflection directly, part of the diffraction pattern was recorded with a prolonged counting time. The data are shown in the insert of Figure 9. A small peak is indeed present at $2 \theta=9.05^{\circ}$. This result (which was not analysed statistically) is quite acceptable, considering the poor statistics.

\section{Discussion}

\section{General}

In this section we will pay attention to a few theoretical aspects that are needed in the discussion of the experimental results. We will briefly mention the relationship between the crystal field anisotropy and magnetic interaction in (a) while in (b) a possible mechanism for the spin canting is discussed.

\section{(a) Crystal field anisotropy and magnetic interaction}

It appears that in many Co(II) compounds the anisotropic properties arise from the action of the crystal field rather than from an anisotropy in the interaction. For orthorhombic site symmetry as we have in the title compound, the combined action of spin-orbit coupling and the orthorhombic crystal field results in a splitting of the ${ }^{4} T_{1 g}$ level into six Kramers doublets. The lowest doublet can be described in terms of an effective $S=\frac{1}{2}$ formalism with anisotropic $g$ values. When the energy of the nearest higher energy level is large compared with the exchange constant and the transition temperature, the magnetic properties at low temperatures will be determined by the ground doublet. As can be seen from the heat capacity results these requirements are reasonably well fulfilled. The real spin $S$ can be expressed into the fictious spin $s$ according to:

$$
S_{i}=\frac{1}{2} g_{i}^{s} s_{i}
$$

with $i=x, y, z$. When we suppose that for the real spin the interaction is isotropic, it will thus be anisotropic in terms of the effective spin $s$. The relative values of $g_{i}{ }^{s}$ will now determine which type of interaction is approached.

\section{(b) Spin canting}

Although several canting mechanisms are possible [28-30], the most appropriate one in our case is that given by Silvera et al. [30]. They showed that the anisotropy in the $g$ values can give rise to large canting angles if the axes of the $g$ tensors are tilted with respect to one another. From the ESR results it is clear that this situation is realised in $\mathrm{Co}(\operatorname{trz})_{2}(\mathrm{NCS})_{2}$. Although Silvera et al. derived the canting angle for a two sublattice system, their result will be applicable here, when we consider the layers in the structure as separate two-sublattice systems. We will give the results of their derivation with reference to Fig. 11, in which $x, y, z$ coincide with the crystallographic, $a, b$, and $c$ directions in Co $(\text { trz })_{2}(\mathrm{NCS})_{2}$. The $x_{1}^{\prime} y_{1}^{\prime} z_{1}^{\prime}$ system with $\Theta>0$ coincides with the principal axes of the $g$ tensors of one half of the Co ions in a layer and $x_{2}{ }^{\prime} y_{2}{ }^{\prime} z_{2}{ }^{\prime}$ with $\Theta<0$ corresponds with the other half. Because there is only canting in the $a-b$ plane, $z^{\prime} \equiv z$. The system is described by an isotropic hamiltonian in the true spins, which are written as $S_{i}=\frac{1}{2} g_{i}{ }^{s} s_{i}$, with $i=x^{\prime}, y^{\prime}, z^{\prime}$. By a transformation of $S_{1}$ and $S_{2}$ to the $x_{1}^{\prime} y_{1}^{\prime} z_{1}^{\prime}$ and $x_{2}^{\prime} y_{2}^{\prime} z_{2}^{\prime}$ systems respectively, an anti-

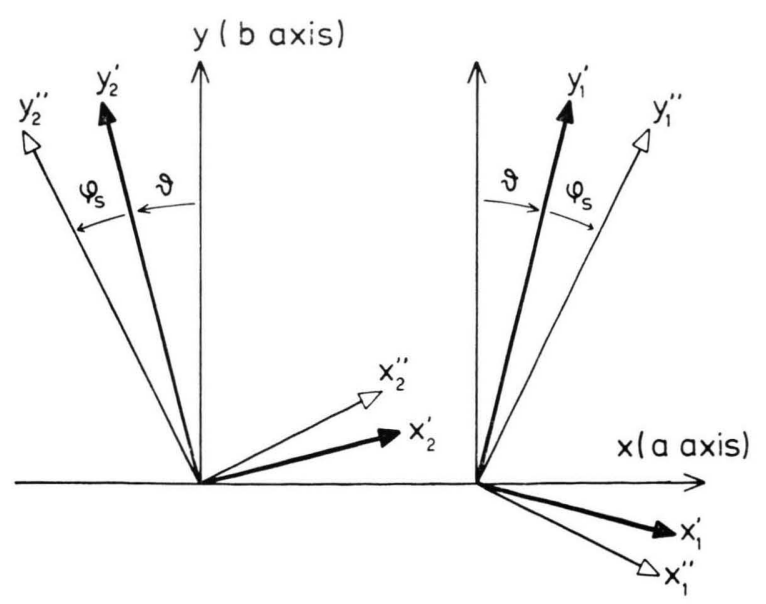

Fig. 11. Coordinate systems referred to in the derivation of the canting angle by Silvera et al. The plane of canting is shown. The $x, y, z$ system coincides with the crystallographic axes, in the $x^{\prime}, y^{\prime}, z^{\prime}$ systems the $g$-tensors are diagonal and in the $x^{\prime \prime}, y^{\prime \prime}, z^{\prime \prime}$ systems $s$ is along $y^{\prime \prime} . z \equiv z^{\prime} \equiv z^{\prime \prime}$ is perpendicular to the plane of drawing. 
symmetric term occurs in the hamiltonian, that gives rise to a canting of $s_{1}$ and $s_{2}$.

Coordinate system $x_{1}^{\prime \prime} y_{1}^{\prime \prime} z_{1}^{\prime \prime}$ and $x_{2}^{\prime \prime} y_{2}{ }^{\prime \prime} z_{2}^{\prime \prime}$ are defined such that below the ordering temperature $\left\langle s_{1}\right\rangle$ and $\left\langle s_{2}\right\rangle$ point along the $y_{1}^{\prime \prime}$ and $y_{2}^{\prime \prime}$ axes. By transformation to these coordinate systems and using the condition that in the $x^{\prime \prime} y^{\prime \prime} \mathrm{z}^{\prime \prime}$ systems $s_{y}$ " must be a constant of the motion, the equilibrium angle $\Phi_{s}$ of the fictitious spin is found to be:

$$
\tan 2 \Phi_{s}=-\frac{2 g_{x^{\prime}}^{8} g_{y^{\prime}}^{8} \tan 2 \theta}{\left(g_{x^{\prime}}^{s}\right)^{2}+\left(g_{y^{\prime}}^{s}\right)^{2}}
$$

The equilibrium direction of the magnetic moments can now be obtained by projecting $s_{1 y^{\prime \prime}}$ onto the $x^{\prime}$ and $y^{\prime}$ axes. The components of the magnetic moment along $x^{\prime}$ and $y^{\prime}$ are then:

$$
\begin{aligned}
& m_{1 x^{\prime}}=\mu_{\mathrm{B}} g_{x^{\prime}} s_{1 x^{\prime}}=\mu_{\mathrm{B}} g_{x^{\prime}} s 1 y^{\prime \prime} \sin \Phi_{s}, \\
& m_{1 y^{\prime}}=\mu_{\mathrm{B}} g_{y^{\prime}} s_{1 y^{\prime}}=\mu_{\mathrm{B}} g_{y^{\prime}} s_{1 y^{\prime \prime}} \cos \Phi_{s} .
\end{aligned}
$$

If $\Phi_{m}$ is the angle between the magnetic moment and $y^{\prime}$, then

$$
\tan \Phi_{m}=\left(g_{x^{\prime}} / g_{y^{\prime}}\right) \tan \Phi_{s} .
$$

\section{Determination of the intralayer exchange constant}

The crystal structure and the heat capacity results suggest that $\mathrm{Co}(\operatorname{trz})_{2}(\mathrm{NCS})_{2}$ may be considered as a 2 -d, $S=\frac{1}{2}$, antiferromagnet. When we assume that the $g$ values found in the ESR experiments on Codoped $\mathrm{Zn}(\operatorname{trz})_{2}(\mathrm{NCS})_{2}$ will not differ very much from those of the bulk compound, the $g$ anisotropy is orthorhombic. Since no calculations on intermediate models are available, we have compared our results with the predictions for quadratic lattices with Ising, $\mathrm{XY}$ and Heisenberg exchange interactions and $S=\frac{1}{2}$.

The susceptibility data in the $b$ direction have been fitted to these models by taking $J / k, g_{b}$ and $\chi_{\text {tip }}$ (temperature independent paramagnetism or Van Vleck term) as adjustable parameters. Figure 4 shows that for $T>T_{c}$ the data can be fitted reasonably well to the XY model [31] $(J / k=-5.0(1) \mathrm{K}$, $g_{b}=5.05$ (10) und $\chi_{\text {tip }}=0.012$ (1) e.m.u. $/$ mole, dashed line) and the Ising model $[32](\mathrm{J} / \mathrm{k}=$ $-5.05(10) \mathrm{K}, g_{b}=5.0(1)$ and $\chi_{\text {tip }}=0.012(1)$ e.m.u./ mole, full line). Comparison with the predictions for the Heisenberg model [33] yielded a less satisfactory result with $J / k=-4.6 \mathrm{~K}$ and $g_{b}=5.1$. As can be seen from Fig. 4 the $\chi_{b}$ vs $T$ data do not extrapolate to the value $\chi_{\text {tip }}$ as one would expect for an easy direction. This deviation may be caused by misorientation, paramagnetic impurities and a canting of the spins away from the $b$ axis (see next section). The $\chi_{c}$ data can be scaled to the $\chi_{b}$ curve for $T>13 \mathrm{~K}$ by taking $g_{c}=4.5$ (see Figure 4 ). The deviations below $T \cong 13 \mathrm{~K}$ may be caused by misorientation, so that there is a contribution from $\chi_{a}$, but it is also possible that the anisotropy in the $b-c$ plane must be ascribed to an anisotropy in both $g$ and the exchange interation. The value of $g_{a}$ can be estimated from the magnetization measurements in the following way. The saturation magnetization in the $a$ and $b$ directions were found as 1.5 and $2.95 \mu_{\mathrm{B}}$, respectively. With $g_{b}=5.0$ and supposing that $g_{a} / g_{b} \cong 1.5 / 2.95$ we find $g_{a} \cong 2.5$. When comparing the three $g$ values: $g_{b}=5.0, g_{c}$ $=4.5$ and $g_{a}=2.5$, the application of the XY model seems to be the best choice, since we have $g_{b} \gtrsim g_{c}$ $\gg g_{a}$.

Next we turn to Fig. 3 in which the heat capacity results are compared with the predictions for the $S=\frac{1}{2}$ Ising [34], XY [35, 36] and Heisenberg [33] models on the quadratic lattice. From the high-temperature part it can be seen that, for $J / k$ $=-5.0 \mathrm{~K}$ as obtained from the susceptibility data, the XY model gives the best agreement with the experimental results. To fit the data to the Ising or Heisenberg curves $J / k$ values would be needed that differ by about $25 \%$ from $-5.0 \mathrm{~K}$ and thus would be incompatible with the results from susceptibility measurements. Closer to $T_{c}$ and below $T_{c}$ the behaviour of $C_{\mathrm{M}}$ is more Ising-like (the same is true for $\chi_{b}$ in Figure 4). The exchange constant $J / k$ is related to the magnetic energy by means of the expression from spin-wave theory [37]:

$$
E / R=z|J| S^{2}\left(1+e_{0} / z S\right) / k
$$

where $z(=4)$ is the number of nearest magnetic neighbours and the quantity $e_{0}$ is a correction arising from zero point spin motions. In Ising systems $e_{0}=0$ and for the quadratic Heisenberg model $e_{0}=0.632$ [37]. No precise value is known for the quadratic XY model, but Metselaar et al. [38] found that $e_{0}$ is approximately 0.4 , with $e_{0}=0.2$ as the lowest possible estimate. By substituting $J / k=$ $-5.0(1) \mathrm{K}$ and $E / R=5.8(2) \mathrm{K}$ into Eq. (3) $e_{0}$ $=0.3(1)$ is calculated, which is in agreement with Metselaar's estimate. 


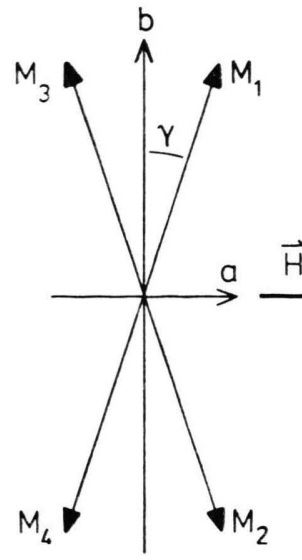

(a) $\mathrm{H}<\mathrm{H}_{\mathrm{C}}^{\mathrm{a}}$

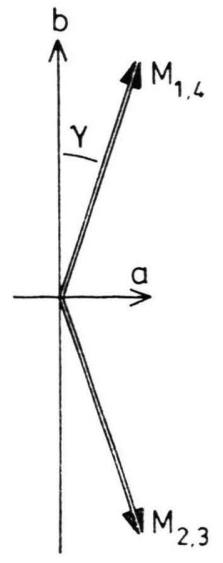

(b) $\mathrm{H}>\mathrm{H}_{\mathrm{C}}^{\mathrm{a}}$
Fig. 12. The four sublattices in the magnetic structure before (a) and after (b) the transition at $H_{\mathrm{c}}$.

The value of $J / k$ can also be determined from the field dependence of the magnetization along the $b$ direction. The curve, depicted in Fig. 8 is very similar to that observed in an uniaxial antiferromagnet with small anisotropy. There are however a number of marked differences. Firstly, the initial slope is fairly high, due to the large value of $\chi_{b}$. Secondly, the linear part of the curve does not extrapolate to zero. Apparently the increase of the magnetization at $38 \mathrm{kOe}$ is less than expected. This can be explained by assuming that the spins flop to a canted arrangement, similar to the one shown in Figure $12 \mathrm{a}$. When we suppose the discontinuity to be a spin-flop transition, the exchange parameter $J_{b} / k$ can be calculated by means of the relation given by Metselaar et al. [38]:

$$
4 z\left|J_{b}\right| S=g_{b} \mu_{\mathrm{B}}\left(H_{c}^{b}+H_{S F}^{2} / H_{c}^{b}\right) .
$$

This formula was derived using an effective field model, taking into account an anisotropic exchange and an anisotropic $g$ factor. With $H_{\mathrm{SF}}=38(2) \mathrm{kOe}$, $H_{c}{ }^{b}=100(5) \mathrm{kOe}$ and $g_{b}=5.0(1)$ we find $\left|J_{b} / k\right|$ $=4.8(3) \mathrm{K}$. Strictly speaking relation $(4)$ is valid only at $T=0$ whereas $H_{\mathrm{SF}}$ and $H_{c}{ }^{b}$ have been determined at $T / T_{c}=0.20$. Besides, Eq. (4) is not fully applicable due to the canting, so that the value of $\left|J_{b} / k\right|$ obtained here must be regarded as an estimate. Nevertheless this value is reasonably in agreement with $J / k=5.0(1) \mathrm{K}$ as found from the heat capacity and susceptibility measurements.

It should be noted that the saturation magnetization along $b$ is $2.95 \mu_{\mathrm{B}}$. That this value is higher than $2.5 \mu_{\mathrm{B}}$, which is the value one would expect with $g_{b}=5.0$, must be attributed to $\chi_{\text {tip }}$. This seems justified, as $\chi_{\text {tip }}=0.012$ e.m.u. $/$ mole (as determined in $H=0$ ) corresponds already with $0.42 \mu_{\mathrm{B}}$ at $200 \mathrm{kOe}$. Furthermore, the contribution of the higher crystal field levels can be expected to increase with the applied magnetic field.

\section{Measurements along a}

The sharp peak in the $\chi_{a}$ vs $T$ curve indicates the existence of weak ferromagnetic moments due to spin canting. The magnetization curves reveal that there is no spontaneous moment in low fields, which is in accordance with the observation that $\chi^{\prime \prime}=0$ in the zero field susceptibilty measurements. Apparently the system is not a weak ferromagnet [39] and hence the peak in the $\chi_{a}$ vs $T$ curve is caused by hidden canting. By combining this information with the observation that the $b$ direction is nearly an easy axis and that no anomalies occur along $c$, we conclude that $\mathrm{Co}(\operatorname{trz})_{2}(\mathrm{NCS})_{2}$ has a magnetic structure in which the magnetic moments are canted away from the $b$ axis and are lying in the $a-b$ plane. These assumptions are corroborated by the neutron diffraction results. The four sublattices comprising the magnetic structure are sketched in Fig. 12 a, where $M_{1}$ and $M_{2}$ belong to one layer and $M_{3}$ and $M_{4}$ to a neighbouring one. Due to the canting, the layers possess a net magnetization component along the $a$ direction, these magnetic moments are antiparallel in adjacent layers. The jump in the $M_{a}$ vs $H$ curve can now be explained by assuming that at the critical field $H_{c}{ }^{a}$ all net moments of the layers become aligned in the direction of the applied field. In order to accomplish this, the direction of half of the spins is reversed (Figure $12 \mathrm{~b}$ ).

Antiferromagnets exhibiting a transition as described here are usually referred to as metamagnets. They have been reviewed recently by Stryjewski and Giordano [40]. The metamagnetic transition between the antiferromagnetic and the weakly ferromagnetic state should be of first order for $0<T<T_{\mathrm{tr}}$ and of higher order for $T_{\mathrm{tr}}<T<T_{c}$. The point on the phase boundary that corresponds with the temperature $T_{\mathrm{tr}}$ is known as the tricritical point. At a first order transition the isothermal susceptibility $\psi_{\mathrm{T}}$ should equal $1 / D(D=$ demagnetizing factor $)$. At $5.0 \mathrm{~K}$ and $906 \mathrm{Oe}, \chi_{\mathrm{T}} \cong 11$ e.m.u./mole is found from the a.c. susceptibility measurements, which is 
much less than $1 / D \cong 80$ e.m.u./mole (estimated from the shape of the sample). Unfortunately it was not possible to determine $\chi_{\mathrm{T}}$ at lower temperatures in this way, due to the long relaxation times. $\chi_{\mathrm{T}}$ can also be determined from the slope of the $M_{a}$ vs $H$ curves. For $T<5.3 \mathrm{~K}$ we find $1 / D \cong 17$ e.m.u./mole. It should be taken into account that the latter measurements were taken on an different sample which besides consisted of several crystals. Nevertheless it seems that the transition is not a true first order one for all temperatures.

The canting angle $\gamma$ of the magnetic moments with respect to the $b$ axis can be calculated from the jump $\Delta M$ in the $M_{a}$ vs $H$ curve, using

$$
\sin \gamma=\Delta M / M_{\mathrm{S}} \text {. }
$$

We will take $\Delta M=0.38(2) \mu_{\mathrm{B}}$, which is the largest value observed and $M_{\mathrm{S}}=2.95(5) \mu_{\mathrm{B}}$, which is the value of the saturation magnetization along $b$. Inserting these values in (5) yields $\gamma=7.4(4)^{\circ}$. From the neutron diffraction results (see Table 1), $\gamma=8^{\circ}$ can be calculated. The agreement with the value obtained above is very good, in view of the large standard deviation of $M_{x}$.

In order to compare these results with Silvera's model we need the tilting angle $\Theta$ of the $g$-tensors and the principal $g$ values. In the ESR experiments it was found that $\Theta=10(2)^{\circ}, g_{x^{\prime}}=2.34(5)$ and $g_{y^{\prime}}=5.42(5)$. Although the local environments of the metal ions in the $\mathrm{Zn}$ and Co compounds differ somewhat, they agree in that the site symmetries are identical and that the tilting angles of the $\mathrm{CoN}_{6}$ and $\mathrm{ZnN}_{6}$ octahedra are equal [8]. We therefore assume that the above mentioned values will be good approximations for Co $(\operatorname{trz})_{2}(\mathrm{NCS})_{2}$. By putting $g_{x^{\prime}} / g_{y^{\prime}} \cong g_{x^{\prime}}^{S} / g_{y^{\prime}}^{S}$ Eqs. (1) and (2) yield $\Phi_{s}=-7.4^{\circ}, \Phi_{m}=-3.2^{\circ}$, so that the canting angle $\gamma=\Theta+\Phi_{m}=7.8^{\circ}$. Considering the uncertainties in this calculation the agreement with the value of $\gamma$ obtained from the jump in $M_{a} \mathrm{vs} \mathrm{H}$ is satisfactory.

The interlayer exchange coupling can be calculated from the values of $H_{c}{ }^{a}$ and $\Delta M$. The interlayer exchange is given by $-z^{\prime}\left|J^{\prime}\right| S^{2}$, where $z^{\prime}$ is the number of nearest neighbours of a Co ion in adjacent layers and $J^{\prime}$ is the interlayer exchange coupling constant. (Like in Eq. (3) we have ignored a term $\cos 2 \gamma_{s}$, with $\gamma_{s}=\Theta+\Phi_{s}$, the spin canting angle, since it is negligibly small.) By equating the changes in Zeeman energy and the interlayer exchange energy at the transition we have at $T=0 \mathrm{~K}$;

$$
2 z^{\prime}\left|J^{\prime}\right| S^{2}=H_{c}{ }^{a} \Delta M .
$$

With $\Delta M=0.38(2) \mu_{\mathrm{B}}$ and $H_{c}{ }^{a}=1015(10) \mathrm{Oe}$, Eq. (6) yields $z^{\prime}\left|J^{\prime}\right| k=5.3(3) \times 10^{-2} \mathrm{~K}$. As regards $z^{\prime}$, from the discussion of the crystal structure it follows that interlayer exchange takes place via contacts between $\mathrm{NCS}^{-}$groups and trz groups in neighbouring layers, so that $z^{\prime}$ may be as large as eight. Thus the ratio of inter- to intralayer exchange becomes $z^{\prime}\left|J^{\prime}\right| / z|J|=2.6(2) \times 10^{-3}$.

So far we have ignored possible contributions from the dipolar interactions. From Blöte's calculations on $\mathrm{Mn}(\operatorname{trz})_{2}(\mathrm{NCS})_{2}$ [9] we estimate that in $\mathrm{Co}(\operatorname{trz})_{2}(\mathrm{NCS})_{2}$ the dipolar interaction energy is about $-5 \times 10^{-3} \mathrm{~K}$. Comparison of this figure with $-z^{\prime}\left|J^{\prime}\right| S^{2} / k=1.3 \times 10^{-2} \mathrm{~K}$ shows that the dipolar energy contributes significantly to the interlayer interactions.

\section{Conclusions}

$\mathrm{Co}(\operatorname{trz})_{2}(\mathrm{NCS})_{2}$ shows 2 -d properties as may be expected from its crystal structure. From the results of different measurements we conclude that the intralayer exchange constant $J / k=-5.0$ (1) K and that the best description is offered by the XY model, despite the fairly large anisotropy in the $b-c$ plane. Hidden canting is found to be present. From the metamagnic transition occurring along $a$ it is inferred that the canting angle of the magnetic moments is $7^{\circ}$, away from the $b$ direction. This value is found to be in satisfactory agreement with theoretical predictions by attributing the canting to tilting of the $g$-tensors in the $a-b$ plane. The ratio of inter- to intralayer exchange $z^{\prime}\left|J^{\prime}\right||z| J \mid$ is found to be only $2.6 \times 10^{-3}$, which explains the observed 2 -d

\begin{tabular}{llll}
\hline $\begin{array}{l}T=300 \mathrm{~K}, \text { Aba2 } \\
\text { (X-ray single crystal }\end{array}$ & $a=7.801(2) \AA$ & $b=16.331(2) \AA$ & $c=9.738(1) \AA$ \\
diffraction) & & & \\
$T=1.2 \mathrm{~K}, \mathrm{Apb}^{\prime} \mathrm{a} 2^{\prime}$ & $a=7.749(1) \AA$ & $b=16.310(3) \AA$ & $c=9.761(1) \AA$ \\
(neutron powder & $M_{x}=0.4(9) \mu \mathrm{B}$ & $M_{y}=2.9(2) \mu \mathrm{B}$ & $M_{z}=0$ \\
diffraction) & $R_{\text {tot. }}=7.3 \%$ & $R_{\text {profile }}=15.6 \%$ & $R_{\text {weight }}=12.5 \%$ \\
& $R_{\text {nucl. }}=6.8 \%$ & $R_{\text {magn. }}=26.8 \%$ & $\chi^{2}=2.3 \quad \mathrm{G}=0.171$ \\
\hline
\end{tabular}

Table 1. Neutron diffraction results at $1.2 \mathrm{~K} .1 \sigma$ standard deviations as calculated by the least-squares program are given in parentheses. For comparison the axes at room temperature have been included. 
behaviour. Since the 2-d XY model does not possess a transition to long-range order [41], the observed transition at $T_{c}=5.71 \mathrm{~K}$ must be ascribed to deviations from the ideal model. In the present case these are the $g$-value anisotropy in the $b$ - $c$ plane and the weak interlayer interactions. This leads to crossovers from 2-d XY behaviour (for $T>2 T_{c}$ ) to 2-d Ising behaviour (for $T<2 T_{c}$ ), explaining the observed temperature dependences of the parallel susceptibility and the specific heat [42].

[1] L. J. de Jongh and A. R. Miedema, Adv. Phys. 23, 1 (1974).

[2] J. N. McElearny, L. L. Balagot, J. A. Muir, and R. D. Spence, Phys. Rev. B 19, 306 (1979).

[3] M. Inoue and M. Kubo, Coord. Chem. Rev. 21, 1 (1976).

[4] P. W. Carreck, M. Goldstein, E. M. McPartlin, and W. D. Unsworth, Chem. Comm. 1971, 1634.

[5] D. Gonzalez, J. Bartholomé, R. Navarro, F. J. A. M. Greidanus, and L. J. de Jongh, J. de Phys. 39, suppl. 8, 762 (1978).

[6] J. G. Haasnoot and W. L. Groeneveld, Z. Naturforsch. 32 b, 553 (1977).

[7] D. W. Engelfriet, J. G. Haasnoot, and W. L. Groeneveld, Z. Naturforsch. 32 a, 783 (1977).

[8] D. W. Engelfriet, W. den Brinker, G. C. Verschoor, and S. Gorter, Acta Cryst. B 35, 2922 (1979).

[9] D. W. Engelfriet and W. L. Groeneveld, Z. Naturforsch. 33 a, 848 (1978)

[10] S. Foner, R. B. Frankel, W. M. Reiff, B. F. Little, and G. J. Long, Solid State Comm. 16, 159 (1975).

[11] A. Herweijer, W. J. M. de Jonge, A. C. Botterman, A. L. M. Bongaarts, and J. A. Cowen, Phys. Rev. B 5, 4618 (1972)

[12] A. C. Botterman, Thesis, Eindhoven 1976.

[13] G. A. Langlet, J. Appl. Cryst. 5, 66 (1972).

[14] D. W. Engelfriet and G. C. Verschoor, to be published.

[15] F. W. Klaaijsen, Thesis, Leiden 1974.

[16] H. T. Witteveen, Thesis, Leiden 1974.

[17] J. W. Arbouw, Thesis, Leiden 1974.

[18] A. van der Bilt, Thesis, Leiden 1978.

[19] H. A. Jordaan, R. Wolf, and D. de Klerk, Physica 69, 129 (1973).

[20] G. M. Nap, in Ref. [9], p. 854.

[21] J. W. Stout and E. Catalano, J. Chem. Phys. 23, 2013 (1955).

\section{Acknowledgements}

The authors wish to thank Mr. J. F. Strang of the Energieonderzoek Centrum Nederland for recording the neutron diffraction data, Drs. P. J. J. M. van der Put of the Technische Hogeschool Delft for his help with the ESR measurements, Dr. L. J. de Jongh and Dr. A. J. van Duyneveldt for discussions and a critical reading of the manuscript and Prof. Dr. I. F. Silvera for a discussion on canting.

[22] R. L. Carlin and A. J. van Duyneveldt, Magnetic Properties of Transition Metal Compounds, SpringerVerlag, New York 1977.

[23] W. Opechowski and R. Guccione, in Magnetism, Vol. 2 A, Acad. Press, New York 1965.

[24] D. W. Engelfriet, to be published.

[25] H. M. Rietveld, J. Appl. Cryst. 2, 65 (1969).

[26] R. E. Watson and A. J. Freeman, Acta Cryst. 14, 27 (1961).

[27] G. E. Bacon, Compilation 1977.

[28] T. Moriya, Phys. Rev. 117, 635 (1960).

[29] T. Moriya, Phys. Rev. 120, 91 (1960).

[30] I. F. Silvera, J. H. M. Thornley, and M. Tinkham, Phys. Rev. 136, A 695 (1964).

[31] L. J. de Jongh, D. D. Betts, and D. J. Austin, Solid State Comm. 15, 1711 (1974).

[32] M. F. Sykes and M. E. Fisher, Physica 28, 919 (1962).

[33] G. A. Baker Jr., H. E Gilbert, J. Ever, and G. S. Rushbrook, Phys. Letters 25 A, 207 (1967).

[34] L. Onsager, Phys. Rev. 65, 117 (1944).

[35] D. D. Betts, C. J. Eliott, and R. V. Ditzian, Can. J. Phys. 49, 1327 (1971).

[36] P. Bloembergen, Physica 85 B, 51 (1977).

[37] F. Keffer, Handbuch der Physik, Vol. 18 Pt. II, Springer-Verlag, Berlin 1966.

[38] J. W. Metselaar, L. J. de Jongh, and D. de Klerk, Physica 79 B, 53 (1975).

[39] H. A. Groenendijk, A. J. van Duyneveldt, and R. D. Willett, to be published in Physica.

[40] E. Stryjewski and N. Giordano, Adv. Phys. 26, 487 (1977).

[41] N. D. Mermin and H. Wagner, Phys. Rev. Letters 17, 1133 (1966).

[42] L. J. de Jongh and H. E. Stanley, Phys. Rev. Letters 36, 817 (1976). 